\title{
Intensive care nursing Himalayan style
}

Sharon M Wetzig RN, BN, GCN (Critical Care), MEd (Further Education and Training), Nurse Educator, Intensive Care Unit, Princess Alexandra Hospital, Brisbane, Queensland, Australia

E-mail: sharon_wetzig@health.qld.gov.au

Key words: developing country intensive care nurse education Nepal

\section{SUMMARY}

* This article provides a personal account of an intensive care nurse's experience of working in intensive care in Nepal.

* Background information is included and several themes are discussed, including:

Initial culture shock: the challenge of adjusting to working in an intensive care environment in a developing country; The role of the International Nepal Fellowship in healthcare development; Examples of the types of intensive care patients admitted to intensive care units in Nepal; Resources and costs: an outline of the issues experienced with limited healthcare resources, multiple re-use of disposable items, and the cost of healthcare for the patients and their families;

Reliance on family involvement in the provision of patient care;

Educational issues: the preparation and delivery of nursing education in a different cultural context.

\section{INTRODUCTION}

The field of intensive care medicine in developing countries is rapidly evolving as economic and technological advancement continue to impact on healthcare (Mustafa 2004). Recently, I was fortunate to have the opportunity to work in an intensive care unit (ICU) in one such country: Nepal. My experience, like that of many others in similar positions, can be described as evoking a 'kaleidoscope of emotions' (Owttrim, 2001). However, through this experience I have learned many valuable lessons, not least of which is the importance of the basics in ICU practice.

In April 2003, I was granted a leave of absence from my position as an ICU nurse educator at the Princess Alexandra Hospital in Brisbane, Australia. During this time, my husband and I spent five months working in Nepal with an aid agency called International Nepal Fellowship (INF). This article describes my personal experience and impressions.

\section{BACKGROUND}

Nepal is a small, landlocked country situated between India and
China with a population of approximately 27 million people. It boasts eight of the world's ten highest peaks and is rich in natural beauty. However, it is also one of the poorest and least developed countries in the world. Current estimates are that at least $42 \%$ of the population lives below the poverty line (Central Intelligence Agency, 2004). Concerns regarding security, with the ongoing Maoist insurgency and terrorist attacks in the United States, have led to a decrease in tourism, a key source of foreign exchange. In addition to poverty and civil conflict, Nepali people face significant hardships in life as a result of the remoteness of many areas and the high incidence of natural disaster. The role of the international community in providing funding for more than $60 \%$ of Nepal's development budget and more than $28 \%$ of total budgetary expenditure is likely to continue (Central Intelligence Agency, 2004).

A comparison of health statistics between Nepal and Australia provides some stark contrasts. For example, in Australia healthy life expectancy is 71 years for males and 74 years for females, while in Nepal the figures are over 20 years less. Also, the child mortality figures in Australia are as low as 6 and 5 per 1,000 for males and females respectively, while Nepal has 81 and 87 per 1,000 (World Health Organisation, 2004).

\section{Role of International Nepal Fellowship}

INF is a Christian non-Government organisation that has been working in the country for over 50 years; in health and development work with the Government, other non-Government organisations, and local communities. This organisation was one of the first to enter Nepal when restrictions on foreigners entering the country were relaxed in 1951. At that time, there were no hospitals in the country, let alone any ICUs. Dramatic changes have been made in a relatively short period of time.

INF's work in Nepal includes provision of hospital and rehabilitation services for people with disabilities, health and development programmes with marginalised communities, medical and surgical camps, HIV/AIDS care, work among displaced people, and secondment of personnel into Government institutions to provide health services training (INF, 2003).

\section{Western Regional Hospital, Pokhara}

I was seconded to Western Regional Hospital (WRH) in Pokhara to provide training for nurses in the ICU. Pokhara is the second largest metropolitan centre in Nepal after Kathmandu. The city is 
about $180 \mathrm{~km}$ north-west of Kathmandu, but travelling time by road (on a good day) is about 10 hours.

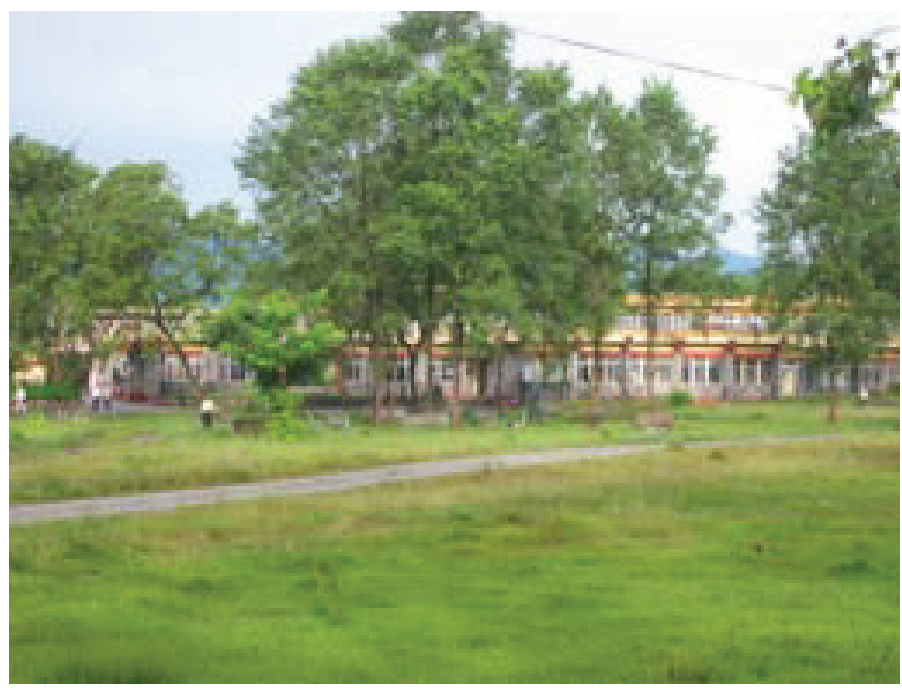

Picture 1: Western Regional Hospital, Pokhara, Nepal.

WRH was built in the 1970s as a 'zonal' hospital (see Picture 1). It currently has around 210 beds, 150 nursing staff and 45 doctors. It is the referral centre for the region, which has a population of somewhere between five and ten million people, although for those who do not live within the metropolitan area, healthcare is neither affordable nor accessible.

The ICU has four beds and receives admissions from the emergency department, the operating theatre and ward areas (see Picture 2). The majority of patients are cardiac/medical patients. Other admissions are from general surgical and obstetric/gynaecological areas. According to the statistics gathered over the last three years, the ICU bed occupancy is $80-90 \%$ and the mortality rate ranges from $20-50 \%$.

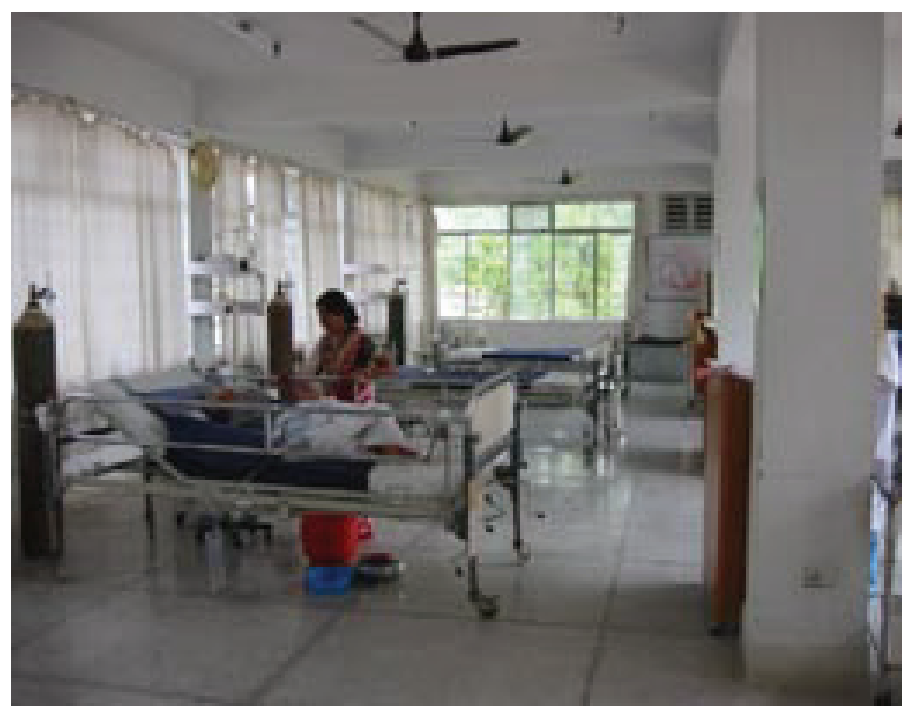

Picture 2: Intensive care unit, Western Regional Hospital.

The ICU medical staff mostly comprises cardiologists and physicians. Anaesthetists are called on to assess and treat patients requiring mechanical ventilation. Surgical and gynaecological patients are treated by their own specialists while in the ICU.

\section{Nursing in Nepal}

In Nepal, nursing is an exclusively female profession. The average daily wage is 200 Nepali Rupees (NR) with some small shift allowances. This is roughly equivalent to a labourer's or housekeeper's daily wage. There are three shifts per day; morning and evening shifts are six hours each and a night shift is 12 hours. The staff have one day off each week and one night off following night duty.

The ICU has ten nursing staff with varying levels of experience and qualification. Until recently, however, none of them had undertaken formal ICU training. The nurse in charge completed an ICU/critical care unit (CCU) training course at a teaching hospital in Kathmandu while I was at WRH. This was funded by INF as part of the agency's desire to provide formal training for Nepali hospital staff.

\section{Culture Shock}

I experienced some culture shock in adjusting to the hospital environment in Pokhara. I have worked in ICU at a large tertiary referral hospital for the past nine years - a highly technological healthcare setting with large amounts of disposable resources with a high value placed on ensuring evidence-based practice. As I soon learned, WRH was a vastly different world.

Every clinical item was precious in Nepal and the concept of disposable items was uncommon. The only things that were not re-used were gauze and cotton wool; everything else was sterilised and re-used or, in the case of needles, syringes and intravenous (IV) lines, used for the duration of the patient's hospitalisation. For example, gloves (regardless of the purpose they were used for) were washed after use, hung up to dry, re-powdered, re-folded and sent to be sterilised (see Picture 3).

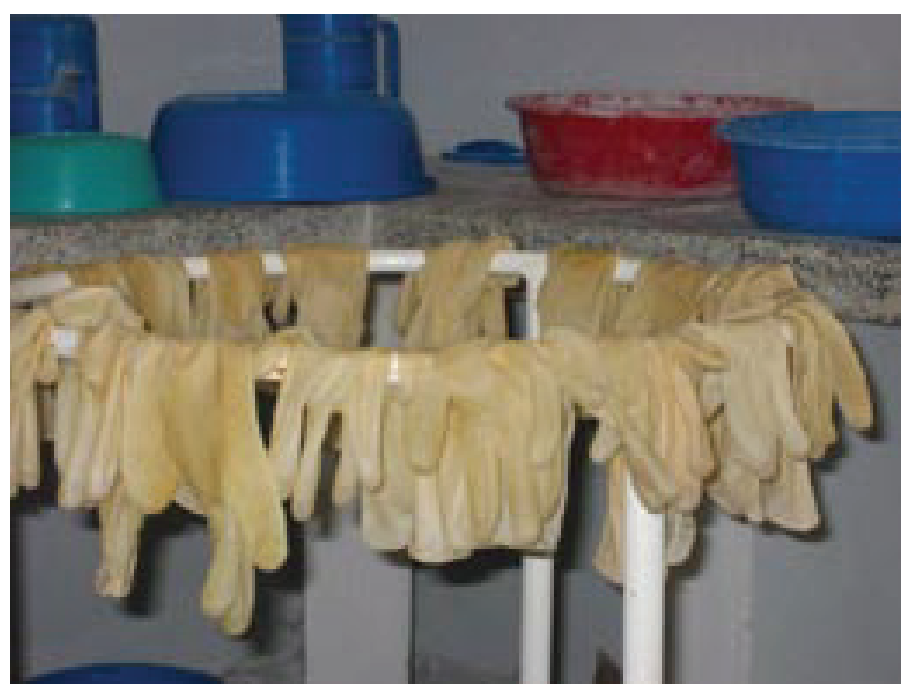

Picture 3: Drying stage of re-sterilisation process for gloves.

The limitations with resources became obvious one day while I was showing one of the newer nurses how to assess a patient with an endotracheal tube. As I began talking about the importance of checking the markings on the tube to assess the depth of insertion, I realised that the tube had been used and re-sterilised so many times that the markings had worn off!

Resource issues were difficult to adjust to, and, not being famil- 
iar with such practices, it was difficult to know if acceptable standards were being met (for example, how many times a disposable item could be re-used and which methods of sterilisation were appropriate). It was quite a surprise for me, one day, to discover a book in the unit containing the WHO standards for re-sterilisation of disposable items, specifically written for hospitals in developing countries.

I could easily have felt overwhelmed by these issues, but when I considered that the hospital was often without power and/or water supply, I realised that there were more important matters to be concerned about. When I visited the laundry, I discovered that all the hospital linen was hand-washed. If the water supply ran out, linen was taken down to the river to be washed!

Even with my initial culture shock, I was continually impressed by the standards of nursing care, given the resources available. One patient was sedated and ventilated for 18 days without access to any pressure relieving devices and didn't develop any pressure sores. The nurses were all very welcoming and keen to learn, and were extremely compassionate and caring people. They were quite open to changes in practice, particularly when they could understand the rationales. There was also very good teamwork within the unit - a valuable feature of any critical care environment (Mustafa, 2004).

\section{Family Involvement in Care}

As part of any hospital admission in Nepal, ICU care includes a high degree of family involvement in basic care. The family were responsible for washing, clothing, feeding and assisting with toileting for patients. Open visiting was not only encouraged but expected. This appeared to be comforting for the patients and provided a sense of familiarity. There may be much to learn from colleagues in developing countries about family-centred care in ICU - a topic of recent interest in many western ICUs (Hammond, 2002).

\section{Cost of Care}

In Nepal, limited Government funding for healthcare means patients are required to pay for each item and service that is used. When a patient was admitted to ICU, we would write a shopping list for the family with all the prescribed medications, needles and syringes, IV lines, electrocardiogram dots etc. and they would purchase these things from the local pharmacy. The ICU was well set up with supplies, so drugs and treatment required in emergency situations could be given and the family could reimburse the unit, but at the ward level, treatment was not given until the needed supplies arrived.

During my time in the hospital, I recorded the cost of various items (see Figure 1) and soon understood why poorer people often do not access healthcare, particularly if these costs are considered in light of a daily wage of around NR200. The cost of 'shopping list' items was variable but added considerably to these costs.

The cost of ICU care is prohibitive for many. Those admitted to ICU needed to be quite rich or have access to family savings. Private health insurance is not an option! Another of INF's activities within the hospital was to administer a 'poor fund' to make money available to those who were unable to afford hospital care. However, a patient in ICU for several days would use this limited resource very rapidly, so the fund was generally not used for ICU patients. This issue added to my culture shock as I have always worked in the public hospital sector in Australia where care is not limited by patients' financial resources.

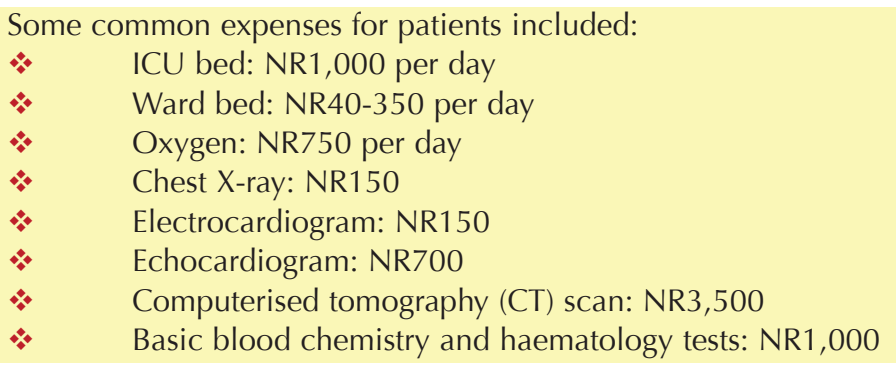

Figure1: Common expenses for patients at the Western Regional Hospital.

Some diagnostic services were very expensive yet they seemed to be used quite frequently in ICU, at times without any obvious clinical benefit. Many patients underwent routine testing but few changes in treatment were made according to results. For example, patients were sent for head CT scans (available in private clinics) but the hospital offered few options for treatment of abnormal findings. Although expensive for the family, it was an exciting experience for me to send an ICU patient (albeit non-ventilated) to have a CT scan in a taxi with only their relatives accompanying them!

\section{Intensive Care Patients}

A number of patients I encountered benefited from intensive care, despite the limited resources. An example was a man with neurocysticerosis (tapeworm infestation in the brain resulting in high intracranial pressures) who required ventilation and seizure management until the treatment took effect. This man would not have survived without ventilation in ICU. Also, many young women who suffered post-partum cardiomyopathy improved markedly with rest and inotrope support. Children admitted with dehydration and/or sepsis benefited from close monitoring during fluid resuscitation.

Other patients' needs were more complex. A teenage boy with severe abdominal sepsis and subsequent adult respiratory distress syndrome required advanced ventilation strategies. However, the ventilator was not sophisticated enough and the medical staff were inexperienced in this area. One of the anaesthetists wanted to find out about ventilation in adult respiratory distress syndrome and I discussed some management strategies with her. However, when it came to altering the treatment, she was reluctant to follow the advice of a foreign, female nurse - three factors that impacted greatly on my status in the ICU. Finally, one specialist recommended giving sodium bicarbonate via the endotracheal tube to 'clear the lungs'. Unfortunately, the patient did not survive.

A great opportunity exists for medical staff to be involved in providing ICU training for anaesthetists and physicians in Nepal. Like many developing countries, Nepal relies heavily on anaesthetists to provide and develop intensive care services (Mustafa, 2004).

\section{Nurse Education}

The training I was asked to provide involved the areas of ventilation and cardiac arrhythmias. However, before I could begin, I had some issues to consider in addition to the language barrier! Teaching and learning in Nepal is very different to what I am used to. Nepali nurses generally follow set routines and procedures 
and do not tend to question treatment decisions or rationales. Culturally, teaching is a one-way process. It can be seen as offensive to question a teacher or to say that you do not understand something, because this implies that your teacher is not very good at their job! This presented some challenges in terms of teaching and learning, and also in the development of critical thinking and problem-solving skills so essential to nursing practice.

I decided to approach training in the unit from an active learning perspective; that is, where learners actively engage in the learning process rather than being passive recipients of information (Theory Into Practice, 2001). For example, rather than providing lectures, I worked with the staff in the clinical area and would discuss the patients we were caring for. I asked questions about disease processes or specific aspects of care and then referred to textbook information together with the staff. This approach worked well and staff enjoyed 'teaching me'. Those with a good grasp of English, or who best understood my attempts at Nepalese, would interpret for the others.

When I left WRH, I presented the nurses with a basic study guide for ICU nursing that I had prepared, based on their requests for information and the general case-mix of the unit. The guide was in

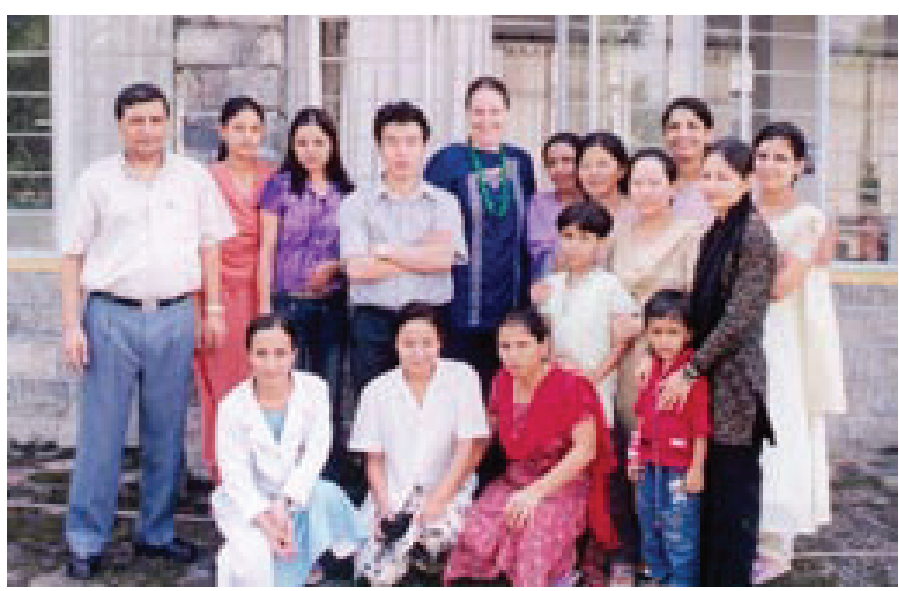

Picture 4: Staff of Western Regional Hospital Intensive Care Unit, 2003 (I am fifth from the left in the back row).

English and included many diagrams, pictures and learning activities that encouraged staff to discuss the topic with other ICU staff.
This was very well accepted by the nursing staff and, for some, it was the first book they had ever personally received.

\section{Conclusion}

The experiences I had while working in Nepal have left me with many happy memories, a great respect and admiration for the healthcare staff working there (see Picture 4), and a renewed appreciation for the many benefits we have in Australian hospitals. Since my return from Nepal, many of my colleagues have shown a keen interest in working in a developing country. If you are interested in this kind of nursing, I would highly recommend it - you will be richly rewarded!

\section{Acknowledgements}

I am so grateful for having been given the opportunity to work in Nepal. I would like to acknowledge my ICU colleagues in Brisbane for releasing me to go and those in Nepal for their friendship, enthusiasm and patience. Thanks also to Gillian who helped me considerably with editing this article.

\section{References}

Central Intelligence Agency. (2004) The World Factbook [online].

Available at: http://www.cia.gov/cia/publications/factbook/geos/ np.html [Accessed 11 March 2005].

Hammond F. (2002) Considering the needs of families in the critical care environment. Australian Critical Care 15 (1), 42-43.

International Nepal Fellowship (INF). (2003) International Nepal Fellowship Website [online]. Available at: http://www.inf.org/assets/ blank.html [Accessed 11 March 2005].

Mustafa I. (2004) Intensive care in developing countries in the Western Pacific. Current Opinion in Critical Care 10 (4), 304-309.

Owttrim A. (2001) Intensive care nursing in Rwanda 1994-1995. Seminars in Perioperative Nursing 10 (3), 126-130.

Theory Into Practice. (2001) Explorations in Learning and Instruction [online]. The Theory Into Practice (TIP) Database, USA Army Research Institute. Available at: http://tip.psychology.org/theories.html [Accessed 11 March 2005].

World Health Organisation (WHO). (2004) The World Health Report 2004 [online]. Available at: http://www.who.int/whr/2004/en/ [Accessed 11 March 2005]. 